\title{
Analysis of the Effect of GEO Satellite Motion on Signal Performance in TWSTFT
}

\author{
Jing Yuejuan ${ }^{1,2,3, a}$, Dong Shaowu ${ }^{1,2, b}$, Li Huanxin ${ }^{1,2, c}$, Zhang Hong ${ }^{1,2, d}$, \\ Yan Min $^{1,2, e}$, Song Huijie ${ }^{1,2,3, f}$, Guang Wei ${ }^{1,2, g}$, Yin Dongshan ${ }^{1,2, h}$ \\ ${ }^{1}$ National Time Service Center, Chinese Academy of Sciences, Xi'an 710600, China \\ ${ }^{2}$ Key Laboratory of Time and Frequency Primary Standards, National Time Service Center, Chinese \\ Academy of Sciences, Xi'an 710600, China \\ ${ }^{3}$ University of Chinese Academy of Sciences, Beijing 100049, China \\ aemail: jingyj@ntsc.ac.cn, bemail: sdong@ntsc.ac.cn, cemail: Ihx@ntsc.ac.cn, ${ }^{\mathrm{d} e m a i l}$ \\ zhong@ntsc.ac.cn, eemail: yanmin_happy@126.com, 'email: \\ songhuijie@ntsc.ac.cn, ${ }^{9} e m a i l:$ guangwei@ntsc.ac.cn, ${ }^{\mathrm{h}} \mathrm{email}$ :yds@ntsc.ac.cn
}

\section{Keywords: Measurement Accuracy; Satellite Motion; Signal Performance; Carrier to Noise Ratio}

\begin{abstract}
For analyzing the effect of GEO(geostationary orbit satellite-GEO) Satellite Motion on Signal Performance in TWSTFT(Two-Way Satellite Time Frequency Transfer), the observation data of TWSTFT and GPS PPP(GPS Precise Point Positioning) which have the similar accuracy between the NTSC (National Time Service Center) and the PTB (Physikalisch-Technishche Bundesanstalt(Germary)) from November 2013 to November 2014 are analyzed. The results show that the motion of GEO satellite has a definite effect on the signal performance, when the satellite used for TWSTFT system starts inclination, the effective observing time is shortened, and the amount of effective observation data is reduced and the data of time difference shows worse agreement with that of GPS PPP, meanwhile, the carrier to noise ratio $(\mathrm{C} / \mathrm{No})$ and the DRMS of time difference of received signal are degraded and there is a negative correlation between C/No with DRMS. The results may be used for the research and analysis of the effect of GEO satellite motion on the measurement accuracy of TWSTFT.
\end{abstract}

\section{Introduction}

Time Keeping and Time Service System offer a solid foundation for the development of national basic technology, and high precision time transfer and synchronization is an important part of the time service [1]. GPS Common-View(GPS CV)、GPS All-in-View (GPS AV)、 two-way satellite time frequency transfer(TWSTFT) and GPS Precise Point Positioning(GPS PPP) are all high accurate mean for remote wireless time transfer technology, and TWSTFT and GPS PPP are the most accurate mean in the world. The long-term stability of TWSTFT is better than that of GPS PPP, while the short-term stability of GPS PPP is better than that of TWSTFT and the accuracy of PPP is very high. Generally, it combines TWSTFT with GPS PPP for High-Precision, the principle diagram of two combing technology is shown in Figure 1.

Laboratory of Time and Frequency Standards of the National Time Service Center(NTSC), the Chinese Academy of Sciences is responsible for establishing and maintaining the national time standard. Atomic time TA(NTSC) and Coordinated Time UTC(NTSC) are realized by precise comparison and calculation of the set of Cesium atomic clocks and Hydrogen atomic clocks, and connected to international time standard by TWSTFT、GPS PPP or GPS AV remote time comparison, which contributes to the calculation of state Coordinated Universal Time(UTC). The signal reception performance of TWSTFT long-distance comparison is affected by the address selection of ground station, the size and quality of antenna, polarization character of feed source, weather, satellite motion and other factors.

Based on the principles of TWSTFT, the paper summarizes the factors that influence the reception intensity and quality of the satellite signal, which is combined with the measured data of 
NTSC from November 2013 to November 2014. Through the data analysis of two kinds of transmission technology of TWSTFT and GPS PPP, it proposes the effects of the satellite motion on the performance of spatial signal transmitted and received in TWSTFT, researches the influence of the C/No of received signal on measurement accuracy of TWSTFT, and which can provide the technical support for improving the international time comparison and traceability of NTSC.

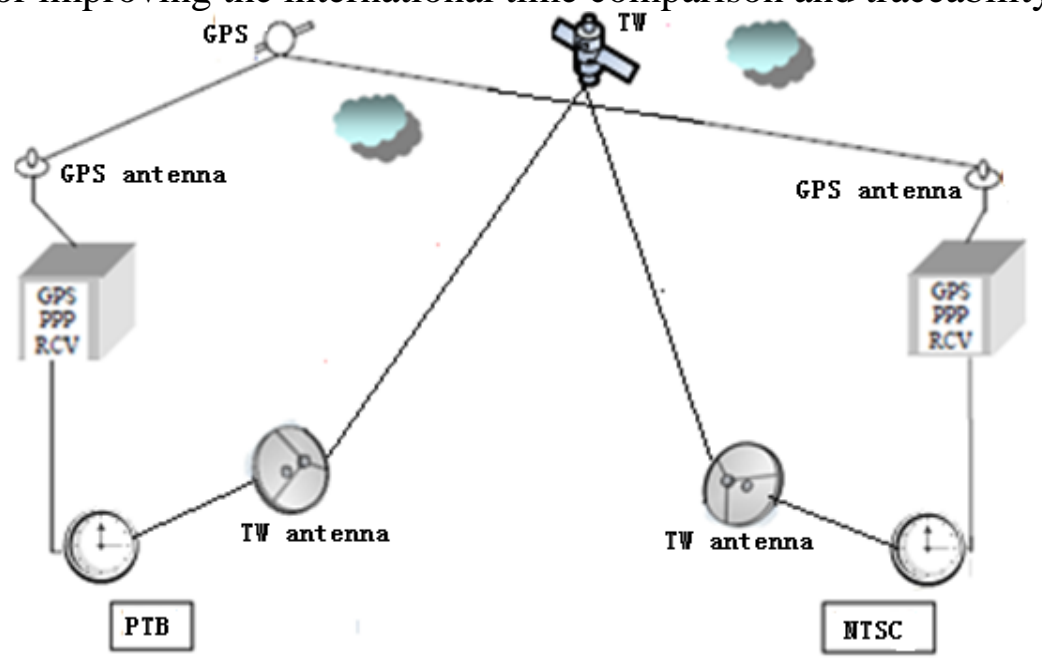

Fig.1. The principle chart of TW and PPP working together

\section{The Influence Factors on Received Signal of TWSTFT}

\subsection{The Principles of TWSTFT}

In theory, it is shown in Figure 2, the paths of transmission and reception of pulses per second between station A and station B are completely identical and the directions of that are opposite, the paths and time delay can completely offset [2], but there are the effects of path delay in transmission, which includes mainly: modem delay, equipment delay of transmission and reception [3]、 satellite transponder delay [4] and delay induced by sagnac effect [5] [6] [7] and so on. The expressions are as follows:

$$
\begin{aligned}
& T I(A)=T A(A)+T A(B)+T T(B)+T C(A)+T S(B)+T D(A)+T R(A) \\
& T I(B)=T A(B)+T A(A)+T T(A)+T C(B)+T S(A)+T D(B)+T R(B)
\end{aligned}
$$

In the type: $T A(k)$ is clock time of station $k$; $T I(k)$ is reading difference of counter of station $\mathrm{k}$; $T T(k)$ is transmission delay which includes Modem delay; $T R(k)$ is receiving delay which includes Modem delay; $T D(k)$ is propagation delay of station $k$; $T S(k)$ is satellite transponder delay of station $\mathrm{k}$; $T C(k)$ is correction of delay induced by sagnac effect of station $\mathrm{k}$. type(1) - type(2):

$$
\begin{aligned}
& \text { TA }(A)-T A(B)=[T I(A)-T I(B)] / 2 \\
& +[T S(A)-T S(B)] / 2 \\
& +[T D(A)-T D(B)] / 2 \\
& +[T T(A)-T R(A)] / 2 \\
& +[T T(B)-T R(B)] / 2 \\
& +[T C(A)-T C(B)] / 2
\end{aligned}
$$

In the type(3): $[T I(A)-T I(B)] / 2$ stands for the reading difference of counter; $[T S(A)-T S(B)] / 2$ stands for the difference of satellite transponder delay; $[T D(A)-T D(B)] / 2$ stands for the difference of propagation delay; $[T T(A)-T T(A)] / 2$ stands for the delay difference between transmission and reception of station $A ;[T T(B)-T T(B)] / 2$ stands for the delay difference between transmission and reception of station $\mathrm{B}$; $[T C(A)-T C(B)] / 2$ stands for the correction of delay induced by sagnac effect.

In the process of TWSTFT, the effects of satellite motion mainly include: One is the change of sagnac effect, the other is the difference of path delay induced by asymmetric up and down paths of signal transmission. The study on the effects of the satellite motion on TWSTFT comparison could 
improve further the measurement accuracy of TWSTFT.

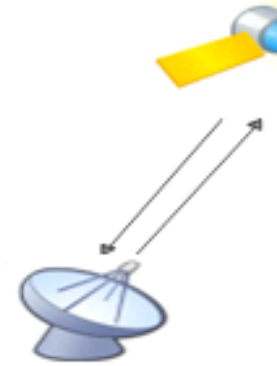

A station

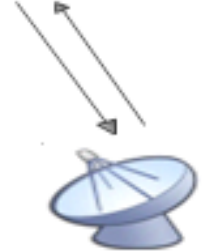

B station

Fig.2. The principle diagram of TWSTFT

\subsection{The Effects of Satellite Motion on Received Signal of TWSTFT}

The key of reception of TWSTFT comparison satellite signal is positioning of receiving antenna, which includes three parameters: azimuth angle of antenna, elevation angle and polarization angle of feed source [8]. In the case of fixed ground station, satellite motion is bound to change the three parameters, which reduces the $\mathrm{C} / \mathrm{No}$ and affects the intensity and quality of received signal.

The carrier to noise ratio (CNR) is one of the important factors that affects the measurement accuracy of TWSTFT and is the ratio of the power and the spectral noise power density of the received signal, where the unit of signal power is $W$, that of spectral density is $\mathrm{W} / \mathrm{Hz}$, therefore, that of $\mathrm{C} / \mathrm{No}$ is $\mathrm{Hz}$, which is expressed for $\mathrm{dBHz}$ in logarithm.

$C / N=10 \lg (P c / P n)$

In the technology of pseudo code spread spectrum, there are many kinds of Pseudo-Noise sequences, which include commonly m sequence, $M$ sequence, GOLD sequence, interceptive $\mathrm{m}$ sequence and so on. One kind of Pseudo-Noise sequence has different measurement accuracy at different code rates [9]. The relation between the C/No and the measurement accuracy of TWSTFT is as follows:

$$
\sigma=\frac{1}{2} T_{C} \sqrt{\frac{d}{T\left(C / N_{O}\right)}}
$$

Where, $T c$ is code-width, $T$ is average time, $d$ is correlator's distance. By the Eq. 5 , we can see that the measurement accuracy $\sigma$ is inversely proportional to the C/No.

The position of the satellite receiving antenna of ground station in TWSTFT system is fixed, due to the influence of many kinds of perturbations, the position of GEO satellite has not absolute rest relative to the observer on earth, and it revolves around a central position to do diurnal motion [10] [11], which results in the error induced by asymmetric up and down paths of signal transmission. Especially when the satellite is at the end of life, it deviates slowly from the normal operational orbit and the motion amplitude becomes more and more that relative to the earth, which is bound to cause the increasing deviation brought about by up and down paths of signal transmission and affect the performance of received signal of the satellite antenna.

\section{Data Analysis}

In order to verify the effects of the satellite motion on the performance of received signal in TWSTFT, the data between TWSTFT and GPS PPP in the laboratory of Time and Frequency Standards of the NTSC from November 2013 to November 2014 is analyzed.

\subsection{The Effects of Satellite Motion on TWSTFT}

\subsubsection{TWSTFT and GPS PPP}

The time difference of TWSTFT and GPS PPP published by the international organization between NTSC and PTB is shown in Figure 3, we can analyze the effects of the satellite motion on TWSTFT transfer technique and take the data of GPS PPP as a reference. 


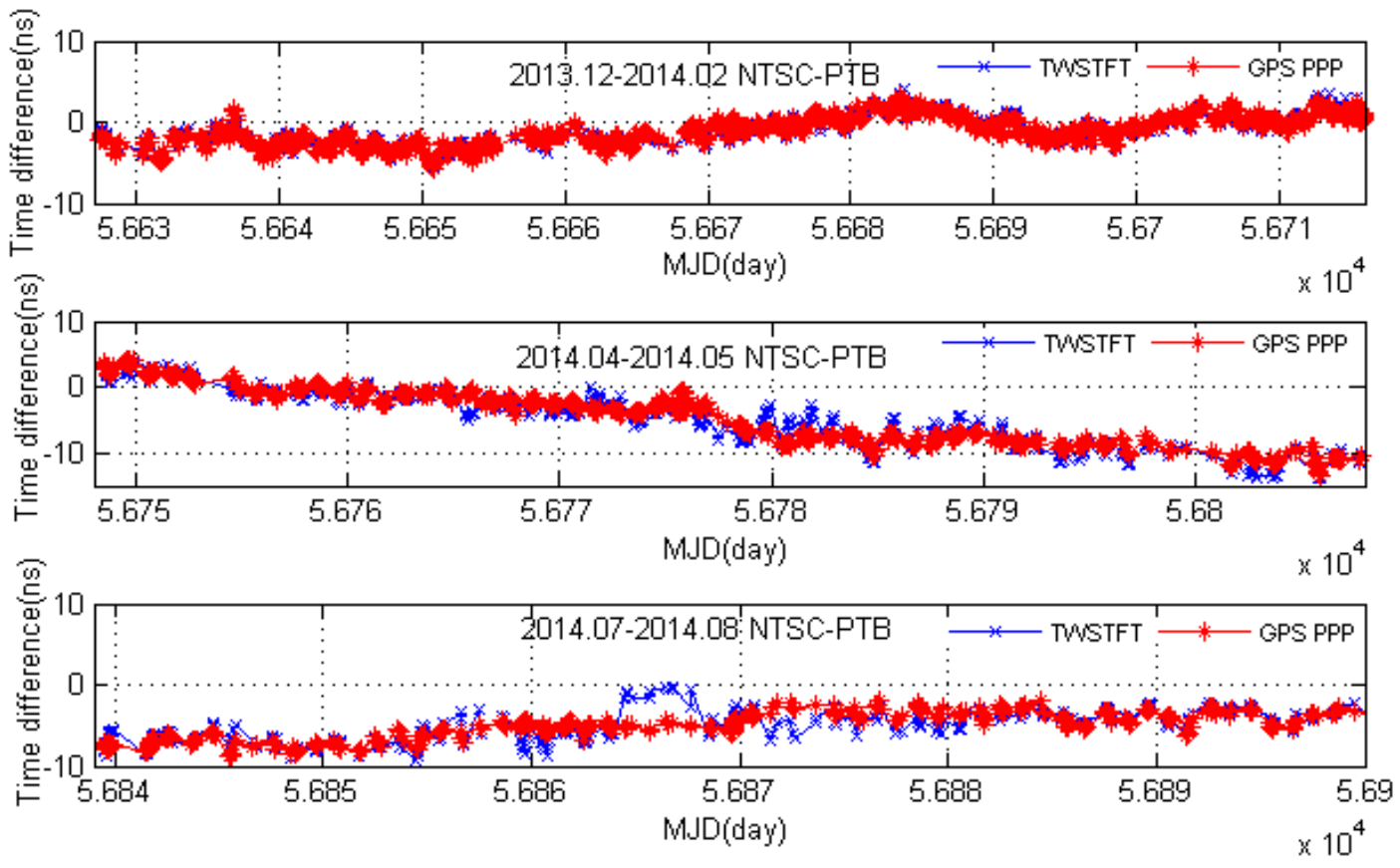

Fig.3. The diagram of the clock difference of TWSTFT and PPP

From Figure 3, we can see that the clock difference data of TWSTFT indicates a good relation with that of GPS PPP from December, 2013 to February, 2014, the clock difference data of TWSTFT shows sustained fluctuation relative to that of PPP from April, 2014 to May, 2014 and the clock difference data of TWSTFT shows a larger fluctuation from July, 2014 to August, 2014. In fact, AM-2 operation started in the inclined orbit from May 2014, deviated from the normal operational orbit and entered gradually the end of life, which is used for TWSTFT comparison between NTSC involved Asia-Pacific laboratories and European laboratories .The TWSTFT system stop running on November 2014, after that, the PPP technology is used for time transfer which connects NTSC involved Asia-Pacific laboratories and International Atomic Time (TAI). From the above analysis, we can get a conclusion that the satellite motion has a certain effect on the performance of TWSTFT comparison and the achieved performance index of time transfer of TWSTFT system is closely related to the performance of the received signal of the satellite antenna.

\subsubsection{Carrier to Noise Ratio and Measurement Accuracy}

(1) The variation of C/No according to the data in TWSTFT system from November 2013 to November 2014 is shown in Figure 4.

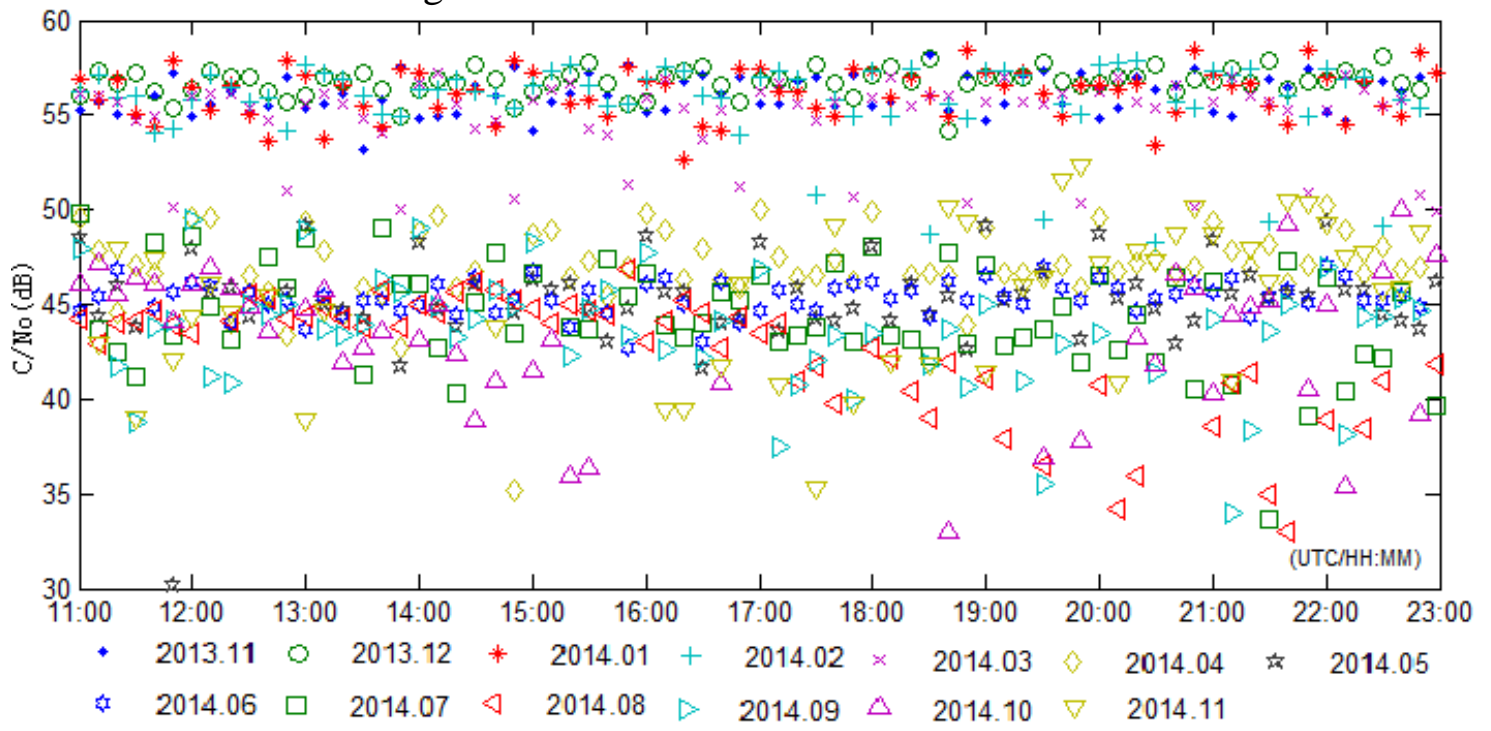

Fig.4. C/No of TWSTFT in November, 2013 November, 2014

The Figure 4 shows C/No values in TWSTFT are above 55dB generally during February, 
2014 March, 2014, and a few data are less than 55dB during November, 2013 March, 2014, the data in April, 2014 November, 2014 are basically lower than 50dB. Under normal condition, the $\mathrm{C} / \mathrm{No}$ value of receiving the signal from each other is more than $55 \mathrm{~dB}$ is valid and good in some fixed stations in TWSTFT system. Therefore, performance index of TWSTFT is excellent during November, 2013 March, 2014, and it is decreased and gets lower and lower as the satellite AM-2's inclination and the end of its life during April, 2014 November, 2014.

(2) Figure 5 and 6 show the data of TWSTFT system which are in the first fifteen-day period of November, 2013 and the first fifteen-day period of November, 2014. According to them, we can analyze the relationships among C/No、DRMS of time difference and the accuracy of the measurement.

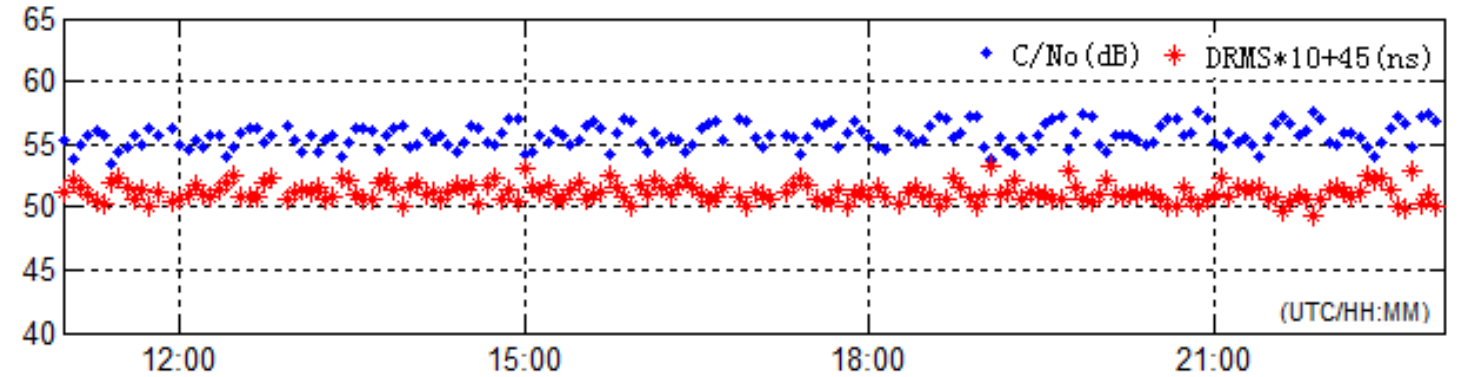

Fig.5. C/No and DRMS of TWSTFT in the first fifteen-day period of November, 2013

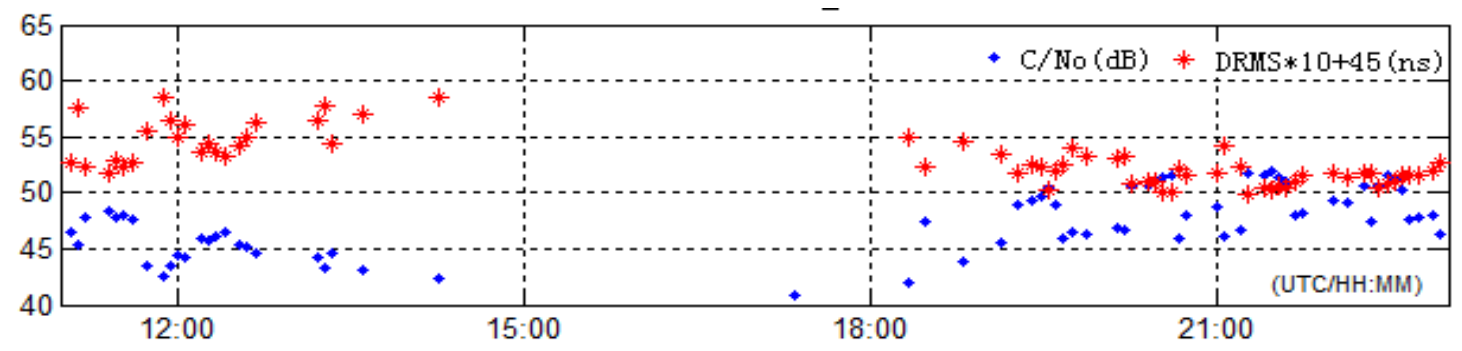

Fig.6. C/No and DRMS of TWSTFT in the first fifteen-day period of November, 2014

From Figure 5 and 6 can be seen, the performance of receiving signal in TWSTFT in the first fifteen-day period of November, 2013 is good, the C/No value is high and stable and above 55Db, and the DRMS is small. In the first fifteen-day period of November, 2014, the quality of receiving signal is poor, the observing time becomes shorter, the amount of effective observation data becomes less, the C/No becomes lower and most of the data value is below $50 \mathrm{~dB}$, the DRMS value is bigger than the first fifteen-day period of November, 2013, according to the Eq. (4) in 2.3, the higher the $\mathrm{C} / \mathrm{No}$ is, the higher the measurement accuracy is. Therefore, satellite operation in the inclined orbit may affect location's variation at earth station (i.e. Azimuth Angle, elevation and polarizing angle of feed), lead to the degradation of the antenna receiving signal level, result in the C/No becoming lower and DRMS value becoming larger, and reduce the measurement accuracy in TWSTFT. Since the degradation of link quality became clear, we stopped the measurement at the end of November, 2014.

\subsection{Correlation Test}

In order to confirm the data consistency of TWSTFT and GPS PPP in equation 3.1.1 and the inverse correlation of variables between signal C/No and DRMS in equation 3.1.2 (2), $t$ test is selected to do normal distribution test and calculate according to equations (6)、(7) and (8). The correlation coefficients between TWSTFT and GPS PPP in some months are listed in table 1, the correlation coefficients between C/No and DRMS in equation 3.1.2 (2) are listed in table 2, the test results are listed in table 3 and table 4 . Table 3 indicates that the data in TWSTF has good agreement with the data of GPS PPP when AM-2 satellite operates normally, and has worse match when AM-2 satellite operation in the inclined orbit started, table 4 indicates that there is inverse correlation between the variables of received signal C/No and DRMS. 
Table 1. Correlation coefficients between PPP and TWSTFT

\begin{tabular}{rc}
\hline Time & Correlation coefficient \\
\hline 2013.12 & 0.957538 \\
2014.01 & 0.980457 \\
2014.02 & 0.952384 \\
2014.04 & 0.885526 \\
2014.05 & 0.846440 \\
2014.07 & 0.611771 \\
2014.08 & 0.456765 \\
\hline
\end{tabular}

Table 2. Correlation coefficients between DRMS and C/No

$$
\begin{aligned}
& \frac{\text { Time }}{\text { 2013.11.01 2013.11.15 }} \text { Correlation coefficient } \\
& r=\frac{\sum(X-\bar{X})(Y-\bar{Y})}{\sqrt{\sum(X-\bar{X})^{2}} \sqrt{\sum(Y-\bar{Y})^{2}}}=\frac{-0.677475}{\left.\sqrt{\left[\sum_{i=1}^{n} x_{i}^{2}-\left(\sum_{i=1}^{n} x_{i} x_{i}\right)^{2} / n\right]\left[\sum_{i=1}^{n} x_{i} \sum_{i=1}^{n} y_{i} / n\right.}-\left(\sum_{i=1}^{n} y_{i}\right)^{2} / n\right]} \\
& t_{r}=\frac{r-0}{S_{r}} \\
& S_{r}=\sqrt{\frac{1-r^{2}}{n-2}}
\end{aligned}
$$

Table 3. Test results of GPS PPP and TWSTF $(\alpha=0.05)$

\begin{tabular}{ccccc}
\hline Time & Degree of freedom & $\begin{array}{c}\text { Correlation } \\
\text { coefficient }\end{array}$ & $\mathrm{t}_{\mathrm{r}}$ & Test result \\
\hline 2013.12 & 331 & 0.957538 & 60.4125 & $\left|\mathrm{t}_{\mathrm{r}}\right|>1.645$, Acceptance test \\
2014.01 & 336 & 0.980457 & 91.3753 & $\left|\mathrm{t}_{\mathrm{r}}\right|>1.645$, Acceptance test \\
2014.02 & 345 & 0.952384 & 58.0015 & $\left|\mathrm{t}_{\mathrm{r}}\right|>1.645$, Acceptance test \\
2014.04 & 330 & 0.885526 & 34.6314 & $\left|\mathrm{t}_{\mathrm{r}}\right|>1.645$, Acceptance test \\
2014.05 & 248 & 0.846440 & 78.8854 & $\left|\mathrm{t}_{\mathrm{r}}\right|>1.645$, Acceptance test \\
2014.07 & 192 & 0.611771 & 10.7159 & $\left|\mathrm{t}_{\mathrm{r}}\right|>1.645$, Acceptance test \\
2014.08 & 186 & 0.456765 & 7.0024 & $\left|\mathrm{t}_{\mathrm{r}}\right|>1.645$, Acceptance test \\
\hline
\end{tabular}

Table 4. Test results of C/No and DRMS ( $\alpha=0.05)$

\begin{tabular}{ccccc}
\hline Time & $\begin{array}{l}\text { Degree of } \\
\text { freedom }\end{array}$ & $\begin{array}{l}\text { Correlation } \\
\text { coefficient }\end{array}$ & $\mathrm{t}_{\mathrm{r}}$ & Test result \\
\hline $2013.11 .01-2013.11 .15$ & 175 & -0.677475 & -12.1844 & $\left|\mathrm{t}_{\mathrm{r}}\right|>1.645$, Acceptance test \\
2014.11.01-2014.11.15 & 66 & -0.877205 & -14.8436 & $\left|\mathrm{t}_{\mathrm{r}}\right|>1.645$, Acceptance test \\
\hline
\end{tabular}

\section{Conclusions and Discussion}

Time Transfer is the process of transmission of time signal in the space, so the quality of the transmitted and received signal directly affects the measurement precision. Under normal conditions, when the C/No value of the receiving signal from each other is more than $55 \mathrm{~dB}$, which is valid and good in fixed stations in TWSTFT, but when satellite operation in the inclined orbit starts and the earth stations and receiving antennas are fixed, which would degrade the strength and quality of the antenna receiving signal, the C/No value is becoming lower and the DRMS value is becoming higher, and decrease the measurement accuracy in TWSTFT. Therefore, when the satellite is in 
Inclination, (1) when the $\mathrm{C} / \mathrm{No}$ is above $40 \mathrm{~dB}$ and below $60 \mathrm{~dB}$, the signal can received; (2) when the $\mathrm{C} / \mathrm{No}$ is between $55 \mathrm{~dB}$ and $60 \mathrm{~dB}$, the signal level is very good;(3) in order to ensure the normal operation of time keeping, when the C/No follows $45 \mathrm{~dB}$, we should stop the measurement using TWSTFT and change the time transfer mode with GPS PPP or others since the degradation of link quality becomes clear.

The study may be offer a reference for researching and analyzing the effect of the signal strength and quality on measurement accuracy, when the satellite motion is in the inclined orbit or time is transferred in the mobile stations, and also have certain significance for the reasonable evaluation of the uncertainty of the final time results in TWSTFT.

\section{Acknowledgement}

In this paper, the research was sponsored by the National Natural Science Foundation of China (Project No. 11473029).

\section{References}

[1] Wang Zhendong. A Research on data processing method of TWSTFT [D]. National Time Service Center, the Chinese Academy of Sciences, 2009.

[2] Michito Imae etc. Two-way satellite time and transfer network in pacific rim region [J].IEEE transactions on instrumentation and measurement.2001, 50(2):559-562.

[3] Li Zhigang, Cheng Zongyi, Feng Chugang, et al. A study of prediction models for ionosphere [J].Chinese J.Geophys. (in Chinese), 2007, 50(2):327-337

[4] Zhang Hong, Li Huanxin, Li Zhigang Temperature Effect of TWSTFT. Journal of Astronautic Metrology and Measurement, 2006.2, 26(1):24-28.

[5] ASCARRUNZ F G, JEFFERTS S R, PARKER T E, etc. Earth station errors in two-way time and frequency transfer [J]. IEEE transactions on instrumentation and measurement, 1997, 46(2): 205-208.

[6] Wang Xiaohan, Yang Xuhai. Calculation of sagnac effect in two way satellite time and frequency transfer [J]. Chinese Journal of Scientific Instrument,

[7] SU Chingchuan. Reinterpretation of the Michelson Morley experiment based on the GPS Sagnac correction [J]. Europhys Lett, 2001, 56 (2):170-174. 27(6) (Suppl.):628-630, 2006.

[8] Chang Juzhen. The parameters of satellite receiving antenna calculated by Excel and the technique of adjusting satellite skills. Cable TV Technology satellite broadcasting, 2008, 9:35-36.

[9] Wu Wenjun. A Research on Errors in Two-way Satellite Time and Frequency Transfer [D].Xian: National Time Service Center, the Chinese Academy of Sciences, 2012.

[10] Schildknecht T, Musci R, Ploner M, et al. Optical observations of space debris in GEO and in Highly-eccentric orbits [J] . Advances in Space Research, 2004, 34: 901-911.

[11] Musci R, Schildknecht T, Ploner M. Analyzing long observation arcs for objects with high area-to-mass ratios in geostationary orbits [J].Acta Astronautica, 2010, 66: 693-703. 